\title{
Risk factors for epithelial ovarian cancer in Japan - results from the Japan Public Health Center-based Prospective Study cohort
}

\author{
ELISABETE WEIDERPASS ${ }^{1,2,3,4}$, SVEN SANDIN $^{1}$, MANAMI INOUE ${ }^{5}$, TAICHI SHIMAZU ${ }^{5}$, MOTOKI IWASAKI ${ }^{5}$, \\ SHIZUKA SASAZUKI $^{5}$, NORIE SAWADA ${ }^{5}$, TAIKI YAMAJI ${ }^{5}$ and SHOICHIRO TSUGANE $^{5 *}$ \\ ${ }^{1}$ Karolinska Institutet, Department of Medical Epidemiology and Biostatistics, PO Box 281, 17177 Stockholm, \\ Sweden; ${ }^{2}$ The Cancer Registry of Norway, PO Box 5313, Majorstuen 0304 Oslo; ${ }^{3}$ Department of Community \\ Medicine, University of Troms $\emptyset$, Troms $\varnothing$, Norway; ${ }^{4}$ Folkhälsan Research Center, Samfundet Folkhälsan, \\ PO Box 211, 00251 Helsinki, Finland; ${ }^{5}$ Epidemiology and Prevention Division, Research Center for Cancer \\ Prevention and Screening, National Cancer Center, 5-1-1 Tsukiji Chuo-ku, Tokyo 104-0045, Japan
}

Received June 2, 2011; Accepted July 4, 2011

DOI: $10.3892 /$ ijo.2011.1194

\begin{abstract}
The aim of this study was to evaluate the risk factors for invasive primary epithelial ovarian cancer among Japanese women. In 1990-1994, 45,748 women aged 40-69 years were enrolled in the Japan Public Health Center-based Prospective Study cohort. Only 86 epithelial ovarian cancer cases were diagnosed during follow-up through 2008, reflecting the low ovarian cancer incidence rates in Japan. Cox proportional hazard models were used to estimate hazard ratios (HR) and 95\% confidence intervals (CI) according to the exposure of interest. The median age at epithelial ovarian cancer diagnosis was 59 years, with a median follow-up before diagnosis of 7.6 years. There were no statistically significant associations for age at menarche or first birth, breastfeeding, use of exogenous hormones, menopausal status at cohort enrollment, height, body mass index, smoking status, second-hand smoke, alcohol consumption, physical activity and family history of cancer in a first-degree relative. The linear decrease in HR associated with each additional birth was 0.75 (95\% CI 0.56-0.99). Among women who usually slept $>7 \mathrm{~h}$ per day, an HR of 0.4 (95\% CI 0.2-0.9) emerged compared to those who slept $<6 \mathrm{~h}$. This study did not confirm risk factors for epithelial ovarian cancer among Japanese women that have been reported in studies carried out elsewhere. Usual sleep duration of $>7 \mathrm{~h}$ per day was inversely associated with epithelial ovarian cancer risk, which is a novel finding that needs to be confirmed in other studies.
\end{abstract}

Correspondence to: Dr Manami Inoue, Epidemiology and Prevention Division, Research Center for Cancer Prevention and Screening, National Cancer Center, 5-1-1 Tsukiji Chuo-ku, Tokyo 104-0045, Japan E-mail:mnminoue@ncc.go.jp

"For the Japan Public Health Center-based Prospective Study Group

Key words: ovary, neoplasm, women, Japan, cohort studies

\section{Introduction}

Ovarian cancer incidence and mortality rates in women in Japan have been increasing in the past decades, as indicated by the National Cancer Center in Tokyo (http://ganjoho.jp/ public/statistics/backnumber/2010_en.html). Ovarian cancer incidence rates in Japan (8.1 per 100,000) (1) are higher than worldwide rates, but relatively low compared to rates in Europe and the United States $(2,3)$. Age-standardized mortality rates of ovarian cancer in Japan increased four-fold, from 0.9 to 3.6 per 100,000 women, between the 1950s and the 1990s (4). In the past two decades they have stabilized, reaching 3.3 per 100,000 women in 2004 (5), which is slightly below the worldwide estimates for that year $(3.8$ per 100,000) (3). Studies on migrants suggest that ovarian cancer incidence rates are higher among Japanese women born in the United States than among those born in Japan (6). The ovarian cancer mortality trends may be explained by birth cohort and period effects in Japan, as mortality has increased within recent birth cohorts for all age groups, with the slope gradually becoming less steep (7).

The historically low rates of ovarian cancer in Japan could offer instructive insights into the etiology of the disease. Individual characteristics, such as reproductive factors [early age at menarche, use of oral contraceptives, late age at menopause, use of estrogen only hormone replacement therapy (HRT), low parity, breastfeeding, age at first and last birth (8-11)], body size (height, weight), lifestyle [smoking (12), alcohol consumption, physical inactivity (13)], and genetic susceptibility (family history of ovarian cancer in a first-degree relative affected at an early age) may be associated with ovarian cancer risk. These associations may differ across populations and ethnic groups, and could partially explain differences in incidence rates across countries. Risk factors for epithelial ovarian cancer among Japanese women have been examined in one prospective cohort study (14-16) besides ours (17), and in several case-control studies $(8,18-25)$, with inconsistent results.

In order to address these issues, we present here data from a prospective cohort study in Japan on the incidence of invasive primary epithelial ovarian cancer in relation to different 
potential risk and protective factors that have been reported or hypothesized to be associated with ovarian cancer risk in Western populations.

\section{Subjects and methods}

The Japan Public Health Center-based Prospective (JPHC) Study, which began in 1990 for cohort I, and in 1993 for cohort II, included Japanese inhabitants living in the municipalities served by 11 different public health centers (henceforth referred to as study areas). Participants were aged 40-59 years in cohort I, and 40-69 years in cohort II at the time of enrollment.

One study area in cohort I was excluded in this report, due to lack of cancer incidence data. An additional 144 women were considered non-eligible because of non-Japanese nationality $(n=20)$, late report of emigration occurring before the start of follow-up ( $n=117)$, incorrect birth date $(n=5)$ or duplicate registration $(\mathrm{n}=2)$. Thus, a population-based cohort of 67,376 women from the JPHC Study were identified as eligible to be included in the present report, 27,377 from cohort I, and 39,999 from cohort II. Of these, 55,840 women [83\%; 22,469 (82\%) in cohort I and 33,371 (83\%) in cohort II] returned the JPHC Study questionnaire, which contained questions on menstrual and reproductive history, use of exogenous hormones (defined as use of any exogenous hormone, including oral contraceptives or HRT), body size (height in $\mathrm{cm}$, and weight in $\mathrm{kg}$ at baseline), smoking habits (including second-hand smoke), alcohol consumption, physical activity during leisure time, usual sleep duration and family history of cancer in a firstdegree relative, among others.

Of the 55,840 women who completed the questionnaire, we excluded from all analyses presented in this article 1,455 women who had a history of cancer at baseline, 8,637 with missing data at enrollment for any of the main exposure variables under study except for alcohol, and 1,340 women due to too many missing values. We further excluded all women who reported artificial menopause before cohort enrollment ( 10\% of the cohort), as there was a possibility that they had been hysterectomized and/or oophorectomized, and therefore not at risk for epithelial ovarian cancer. Information on hysterectomy and oophorectomy was not available. This left a total of 45,748 women who were followed up.

Study participants were informed in writing about the objectives and methods of the JPHC Study and those who responded to the questionnaire were regarded as consenting to participate in the study. The study protocol was approved by the institutional review board of the National Cancer Center, Tokyo, Japan.

Follow-up and identification of cancer cases. Person-years of follow-up were calculated from the date of return of the baseline questionnaire until the date of diagnosis of epithelial ovarian cancer, relocation from the study area (i.e., migration), death, or end of the study period (December 31, 2008), whichever occurred first. Data on residential relocation were obtained from residential registries. Among the 45,748 study subjects followed up, 3,046 died, 4,290 moved and 190 were lost to follow-up. Death certificates, coded according to the requirements of the Ministry of Health, Labor and Welfare, were collected through local public health centers with permission.
Incidence data for cancer were collected though two data sources, major local hospitals and population-based cancer registries. Death certificates were used to supplement the information on cancer incidence. Cancer incidence data were only collected for subjects living in the study areas. Members of the JPHC Study Group coded the tumor site of origin and histological subtype using the International Classification of Diseases for Oncology, 3rd edition. Epithelial ovarian cancer cases were defined as women with an incident cancer with the following codes: C56-9, histologies 8000, 8041, 8120, 8140, 8260, 8310, $8323,8380,8440,8441,8450,8460,8470,8471$ and 8480 . Non-epithelial tumors, namely carcinosarcomas (C56-9, histology 8980, $\mathrm{n}=3$ ), dysgerminomas (C56-9, histology 9060, $\mathrm{n}=1)$ and teratomas $(\mathrm{C} 56-9$, histology $9080, \mathrm{n}=2)$ were excluded from the analysis, as their risk factors may differ from those for epithelial ovarian cancer (26), and the small number of cases in our cohort would have precluded meaningful conclusions. All other histological subtypes were combined in the analyses, as we did not have enough cases to perform separate analyses for each histological subtype. Borderline ovarian tumors are not recorded by the cancer registration system in Japan, and therefore were not included in this study.

Statistical analysis. The stratified Cox proportional hazard model was used to calculate hazard ratios (HR) and $95 \%$ confidence intervals (CI) estimating the relative risk of epithelial ovarian cancer according to the exposures under study. HRs and $95 \%$ CIs for continuous variables are reported with two decimal digits, while all others are reported with one decimal digit only. Attained age with delayed entry was used as a time scale (27) in the model. Treating the study areas as a stratifying variable (28) allowed for different baseline hazards in the different study areas. The assumption of the proportional hazards in the Cox model was checked and found to be satisfactory (28).

We performed analyses based on the following variables: age at menarche $(<14,14,15,>15$ years, and as a continuous variable per 1-year increase), parity (nulliparous versus parous, and $0,1,2,3,>3$ births, and as a continuous variable per additional birth), age at first birth among parous women $(<22$, $22-25,26-29,>29$ years, and as a continuous variable per 1 -year increase), history of breastfeeding among parous women (no, yes) use of exogenous hormones (no, yes; please note that as only information on any hormone use was available, it was not possible to separate use of oral contraceptives or HRT), menopausal status at enrollment (premenopausal, postmenopausal with natural menopause), height (in $\mathrm{cm}$, and as a continuous variable per $10 \mathrm{~cm}$ increase), body mass index (BMI, calculated as weight in $\mathrm{kg}$ at baseline divided by height in $\mathrm{m}^{2}$; the self-reported height and weight data were validated (29) and then categorized: <18.5, 18.5-19.9, 20-22.9, 23-24.9, 25-29.9, >29.9, and as a continuous variable per BMI unit increase), smoking status (never or ever smoker), exposure to second-hand smoke (no, yes), alcohol consumption (no, yes, and as a continuous variable in grams per week), physical activity during leisure time (no, yes), usual sleep duration $(<6$, $6-7,>7 \mathrm{~h}$ per day, and as a continuous variable per hour), and family history of cancer in a first-degree relative (i.e., mother, father, siblings; no, yes). Cut-points for categorization of each variable were decided based both on the hypothesized 


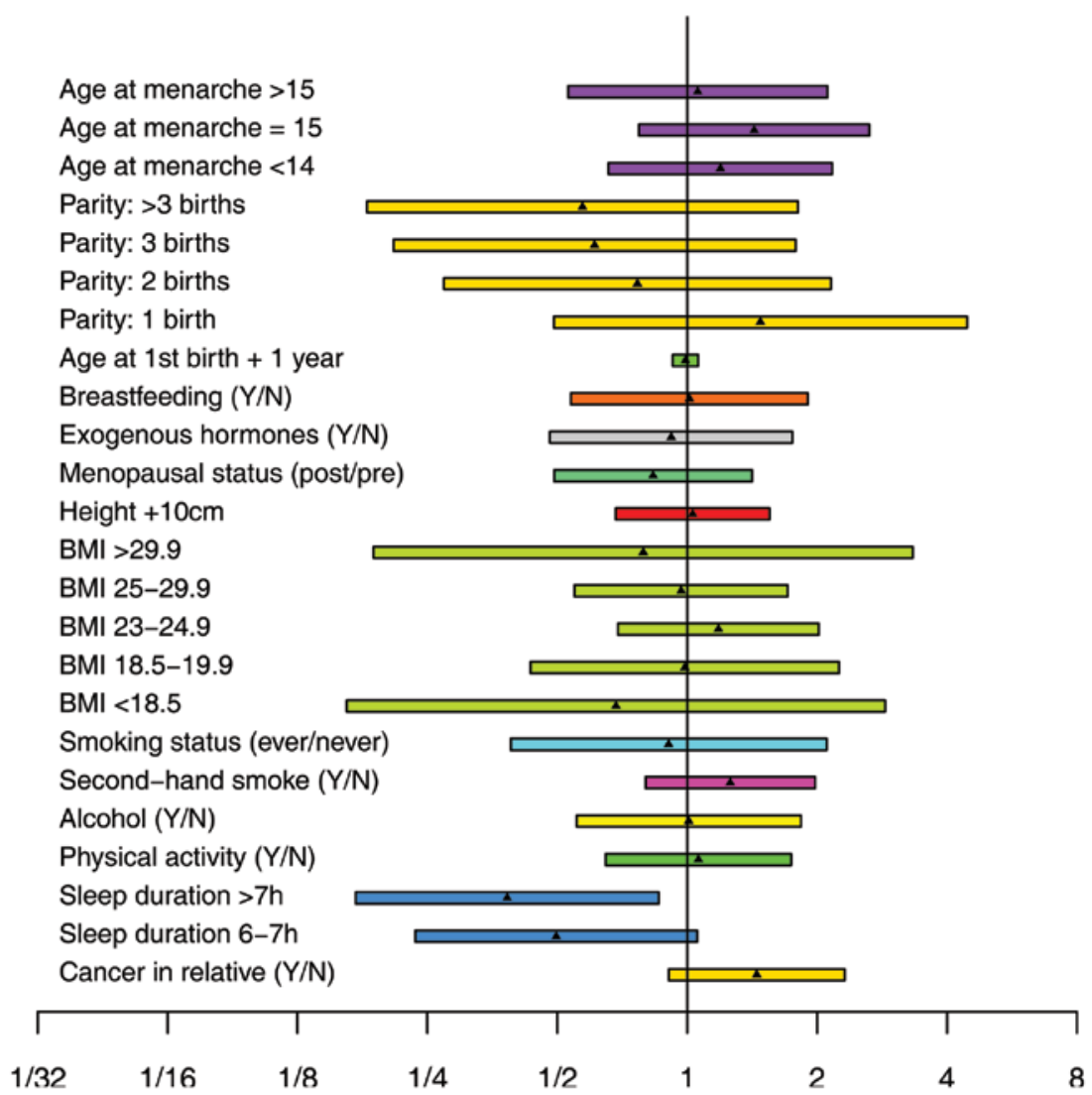

Figure 1. Hazard ratios (black triangles) and two-sided 95\% confidence intervals (horizontal boxes) of epithelial ovarian cancer according to selected menstrual and reproductive factors, anthropometric characteristics, and lifestyle factors. The Japan Public Health Center-based Prospective Study. Abbreviations: BMI, body mass index; Y, yes; N, no. NOTE: Estimates correspond to Table II. Reference categories are: age at menarche, 14 years; parity, 0; breastfeeding, no; exogenous hormones, no; menopausal status, premenopausal; BMI, 20-22.9; smoking status, never; second-hand smoke, no; alcohol, no; physical activity, no; sleep duration, $<6 \mathrm{~h}$; cancer in relative, no.

biological effects on epithelial ovarian cancer risk, as well as on the frequency distribution of the variables within the cohort (17,29-31).

We fitted basic models, further stratifying by study area and using attained age as a time scale. We also fitted multivariable models including all variables as described above simultaneously. To ensure that comparisons between models and covariables were not dependent on missing values, all models were fitted to a dataset with complete data for all the primary covariables of interest. In a supplementary set of analyses, one restricted to parous women and another to women answering the question on alcohol consumption, we also re-fitted the models on datasets using all available data for each model. Second-hand smoke was analyzed among all women, regardless of their smoking status, and subsequently restricted to never smokers.

All p-values reported are two-sided, and the significance level was set at $p<0.05$. For data management and for summary statistics and graphs the SAS software version 9.2 was used. Cox models were fitted using the survival package in the $\mathrm{R}$ software version 2.9.2 running Linux Debian.

\section{Results}

Baseline characteristics of the study population are shown in Table I. The 45,748 women included our analyses were followed up for a median of 16 years. During follow-up, only 86 newly arising cases of epithelial ovarian cancer were recorded: 35 serous adenocarcinomas (histologies 8140, 8260, $8441,8450,8460), 16$ clear-cell adenocarcinomas (histology 8310), 13 mucinous adenocarcinomas (histologies 8470, 8471, 8480), six endometrioid adenocarcinomas (histology 8380), three other adenocarcinomas (small cell adenocarcinoma, histology 8041; transitional adenocarcinoma, histology 8120; other, histology 8323), and 13 adenocarcinomas not otherwise specified (histology 8000). The overall age-standardized incidence rate (SIR) of ovarian cancer in the cohort was 11 per 100,000 women.

The median age at diagnosis was 59 years, with a median follow-up before diagnosis of 7.6 years. There were no differences between the mean ages at enrollment, menarche, or first birth, nor between BMI and usual sleep duration between women with and without epithelial ovarian cancer. The proportions of women who were nulliparous, had never breastfed (among parous women), had used exogenous hormones, were premenopausal at enrollment, and who reported smoking, being exposed to second-hand smoke, consuming alcohol, and being physically active during leisure time were also similar between women who did and did not develop epithelial ovarian cancer during follow-up. The proportion of women with a history of cancer in a first-degree relative was $30.2 \%$ among women with, and $22.7 \%$ among women without epithelial ovarian cancer (Table I). 
Table I. Characteristics of study participants $(n=45,748)$ at baseline. ${ }^{\mathrm{a}}$

\begin{tabular}{|c|c|c|c|c|c|c|}
\hline \multirow[t]{2}{*}{ Characteristics } & \multicolumn{2}{|c|}{ Women } & \multicolumn{2}{|c|}{$\begin{array}{l}\text { Epithelial ovarian } \\
\text { cancer cases }\end{array}$} & \multicolumn{2}{|c|}{ Non-cases } \\
\hline & Number & $\%$ & Number & $\%$ & Number & $\%$ \\
\hline Number of participants & 45,748 & 100 & 86 & 0.2 & 45,662 & 99.8 \\
\hline \multicolumn{7}{|l|}{ Age at enrollment, years } \\
\hline $40-49$ & 19,501 & 42.6 & 34 & 39.5 & 19,467 & 42.6 \\
\hline $50-59$ & 18,848 & 41.2 & 42 & 48.8 & 18,806 & 41.2 \\
\hline $60-69$ & 7,399 & 16.2 & 10 & 11.6 & 7,389 & 16.2 \\
\hline \multicolumn{7}{|l|}{ Age at menarche, years } \\
\hline$<14$ & 13,203 & 28.9 & 26 & 30.2 & 13,177 & 28.9 \\
\hline 14 & 11,208 & 24.5 & 19 & 22.1 & 11,189 & 24.5 \\
\hline 15 & 9,565 & 20.9 & 23 & 26.7 & 9,542 & 20.9 \\
\hline$>15$ & 11,772 & 25.7 & 18 & 20.9 & 11,754 & 25.7 \\
\hline \multicolumn{7}{|l|}{ Parity } \\
\hline Nulliparous & 2,904 & 6.4 & 6 & 7.0 & 2,898 & 6.3 \\
\hline 1 & 3,421 & 7.5 & 12 & 14.0 & 3,409 & 7.5 \\
\hline 2 & 16,231 & 35.5 & 34 & 39.5 & 16,197 & 35.5 \\
\hline 3 & 12,915 & 28.2 & 21 & 24.4 & 12,894 & 28.2 \\
\hline$>3$ & 10,277 & 22.5 & 13 & 15.1 & 10,264 & 22.5 \\
\hline Parous, number of births & 42,844 & 93.6 & 80 & 93.0 & 42,764 & 93.6 \\
\hline \multicolumn{7}{|l|}{ Age at first birth among } \\
\hline \multicolumn{7}{|l|}{42,844 parous women, years } \\
\hline$<22$ & 6,103 & 14.2 & 9 & 11.2 & 6,094 & 14.2 \\
\hline $22-25$ & 20,975 & 49.0 & 41 & 51.2 & 20,934 & 49.0 \\
\hline $26-29$ & 11,982 & 28.0 & 20 & 25.0 & 11,962 & 28.0 \\
\hline$>29$ & 3,784 & 8.8 & 10 & 12.5 & 3,774 & 8.8 \\
\hline \multicolumn{7}{|l|}{$\begin{array}{l}\text { Breastfeeding among } 42,844 \\
\text { parous women }\end{array}$} \\
\hline Nulliparous/never & 2,904 & 6.3 & 6 & 7.0 & 2,898 & 6.3 \\
\hline No & 5,413 & 11.8 & 12 & 14.0 & 5,401 & 11.8 \\
\hline Yes & 37,431 & 81.8 & 68 & 79.0 & 37,363 & 81.8 \\
\hline \multicolumn{7}{|l|}{ Use of exogenous hormones } \\
\hline No & 39,927 & 87.3 & 75 & 87.2 & 39,852 & 87.3 \\
\hline Yes & 5,821 & 12.7 & 11 & 12.8 & 5,810 & 12.7 \\
\hline \multicolumn{7}{|l|}{$\begin{array}{l}\text { Menopausal status at } \\
\text { enrollment }^{\mathrm{b}}\end{array}$} \\
\hline Premenopausal & 19,815 & 43.3 & 40 & 46.5 & 19,775 & 43.3 \\
\hline Postmenopausal (natural) & 25,933 & 56.7 & 46 & 53.5 & 25,887 & 56.7 \\
\hline \multicolumn{7}{|l|}{ Body mass index, $\mathrm{kg} / \mathrm{m}^{2}$} \\
\hline$<18.5$ & 1,654 & 3.6 & 2 & 2.3 & 1,652 & 3.6 \\
\hline 18.5-19.9 & 3,900 & 8.5 & 7 & 8.1 & 3,893 & 8.5 \\
\hline $20-22.9$ & 16,667 & 36.4 & 31 & 36.0 & 16,636 & 36.4 \\
\hline $23-24.9$ & 10,793 & 23.6 & 24 & 27.9 & 10,769 & 23.6 \\
\hline$(20-24.9)$ & 27,460 & 60.0 & 55 & 64.0 & 27,405 & 60.0 \\
\hline $25-29.9$ & 11,302 & 24.7 & 20 & 23.3 & 11,282 & 24.7 \\
\hline$>29.9$ & 1,432 & 3.1 & 2 & 2.3 & 1,430 & 3.1 \\
\hline \multicolumn{7}{|l|}{ Smoking status } \\
\hline Never & 41,957 & 91.7 & 80 & 93.0 & 41,877 & 91.7 \\
\hline Ever & 3,791 & 8.3 & 6 & 7.0 & 3,785 & 8.3 \\
\hline \multicolumn{7}{|l|}{$\begin{array}{l}\text { Exposure to second-hand } \\
\text { smoke among all women }\end{array}$} \\
\hline No & 29,479 & 64.4 & 49 & 57.0 & 29,430 & 64.4 \\
\hline Yes & 16,269 & 35.6 & 37 & 43.0 & 16,232 & 35.6 \\
\hline
\end{tabular}


Table I. Continued.

\begin{tabular}{|c|c|c|c|c|c|c|}
\hline \multirow[t]{2}{*}{ Characteristics } & \multicolumn{2}{|c|}{ Women } & \multicolumn{2}{|c|}{$\begin{array}{l}\text { Epithelial ovarian } \\
\text { cancer cases }\end{array}$} & \multicolumn{2}{|c|}{ Non-cases } \\
\hline & Number & $\%$ & Number & $\%$ & Number & $\%$ \\
\hline \multicolumn{7}{|l|}{$\begin{array}{l}\text { Exposure to second-hand smoke } \\
\text { among } 41,957 \text { never smokers }\end{array}$} \\
\hline No & 27,852 & 66.4 & 47 & 58.8 & 27,805 & 66.4 \\
\hline Yes & 14,105 & 33.6 & 33 & 41.2 & 14,072 & 33.6 \\
\hline \multicolumn{7}{|l|}{$\begin{array}{l}\text { Alcohol consumption among } \\
44,408 \text { women }^{c}\end{array}$} \\
\hline No & 36,707 & 82.7 & 68 & 82.9 & 36,639 & 82.7 \\
\hline Yes & 7,701 & 17.3 & 14 & 17.1 & 7,689 & 17.3 \\
\hline \multicolumn{7}{|l|}{$\begin{array}{l}\text { Physical activity during } \\
\text { leisure time }\end{array}$} \\
\hline No & 34,066 & 74.5 & 64 & 74.4 & 34,002 & 74.5 \\
\hline Yes & 11,682 & 25.5 & 22 & 25.6 & 11,660 & 25.5 \\
\hline \multicolumn{7}{|l|}{ Usual sleep duration, $\mathrm{h}$} \\
\hline$<6$ & 2,419 & 5.3 & 8 & 9.3 & 2,411 & 5.3 \\
\hline $6-7$ & 27,085 & 59.2 & 53 & 61.6 & 27,032 & 59.2 \\
\hline$>7$ & 16,244 & 35.5 & 25 & 29.1 & 16,219 & 35.5 \\
\hline \multicolumn{7}{|l|}{$\begin{array}{l}\text { Family history of cancer } \\
\text { in a first-degree relative }\end{array}$} \\
\hline No & 35,337 & 77.2 & 60 & 69.8 & 35,277 & 77.3 \\
\hline Yes & 10,411 & 22.8 & 26 & 30.2 & 10,385 & 22.7 \\
\hline Mean and SD & Mean & $\mathrm{SD}$ & Mean & SD & Mean & SD \\
\hline Age at enrollment, years & 51 & 7.9 & 51 & 7.5 & 51 & 7.9 \\
\hline Age at menarche, years & 15 & 1.9 & 14 & 1.7 & 15 & 1.9 \\
\hline Age at first birth among & 25 & 3.5 & 25 & 3.6 & 25 & 3.5 \\
\hline 42,844 parous women, years & & & & & & \\
\hline Height, cm & 152 & 5.6 & 152 & 5.4 & 152 & 5.6 \\
\hline Body mass index, $\mathrm{kg} / \mathrm{m}^{2}$ & 23 & 3.2 & 23 & 2.6 & 23 & 3.2 \\
\hline Alcohol consumption, g/week & 17 & 89.2 & 12 & 53.1 & 17 & 89.2 \\
\hline Usual sleep duration, $\mathrm{h}$ & 7 & 1 & 7 & 1 & 7 & 1 \\
\hline
\end{tabular}

${ }^{a}$ The Japan Public Health Center-based Prospective Study. ${ }^{b}$ Women who reported artificial menopause were excluded from the cohort, as the

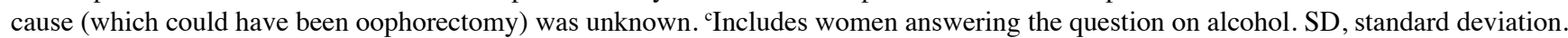

The HRs and 95\% CIs of epithelial ovarian cancer according to potential risk factors are shown in Table II, and displayed graphically in Fig. 1. Overall, there were no statistically significant associations for age at menarche, age at first birth, breastfeeding, use of exogenous hormones, menopausal status at cohort enrollment, height (multivariable HR per $10 \mathrm{~cm}$ increase $=1.03,95 \%$ CI 0.68-1.55), BMI (multivariable HR per BMI unit increase $=1.00,95 \%$ CI 0.94-1.08), smoking, exposure to second-hand smoke, alcohol consumption, physical activity during leisure time and family history of cancer in a first-degree relative (multivariable $\mathrm{HR}=1.4$, 95\% CI 0.9-2.3) (Table II, Fig. 1). The decrease in HR associated with each additional birth was 0.75 (95\% CI 0.56-0.99). In both the ageand study area-adjusted model, as well as in the multivariable model there was evidence of an inverse association with usual sleep duration. Women who usually slept more than $7 \mathrm{~h}$ per day had an HR of 0.4 (95\% CI 0.2-0.9) compared to those who usually slept less than $6 \mathrm{~h}$; results were similar in the model adjusted for age and study area, and in the multivariable model (Table II, Fig. 1). The results described above and presented in Table II remained basically unchanged when we restricted the analyses to parous women, when we included women who reported artificial menopause (data not shown), when we excluded the variable use of exogenous hormones from the multivariate models, and when we used covariables in a continuous format instead of as categorical variables (for age at menarche, parity, age at first birth among parous women, height, BMI, alcohol consumption and usual sleep duration). Results of the supplementary set of analyses, where we re-fit models on datasets using all available data for each model 
Table II. Hazard ratios (HR) and 95\% confidence intervals (CI) of epithelial ovarian cancer according to menstrual and reproductive factors, anthropometric characteristics, and lifestyle factors among study participants $(n=45,748){ }^{a}$

\begin{tabular}{|c|c|c|c|c|}
\hline \multirow[t]{2}{*}{ Characteristics } & \multicolumn{2}{|c|}{ Age and study area adjusted } & \multicolumn{2}{|c|}{ Multivariable adjustment } \\
\hline & $\mathrm{HR}^{\mathrm{b}}$ & $95 \% \mathrm{CI}$ & $\mathrm{HR}^{\mathrm{c}}$ & $95 \% \mathrm{CI}$ \\
\hline \multicolumn{5}{|l|}{ Age at menarche, years } \\
\hline$<14$ & 1.2 & $0.7-2.2$ & 1.2 & $0.7-2.2$ \\
\hline 14 & 1.0 (ref) & & 1.0 (ref) & \\
\hline 15 & 1.4 & $0.8-2.6$ & 1.4 & $0.8-2.6$ \\
\hline$>15$ & 1.0 & $0.5-2.0$ & 1.1 & $0.5-2.1$ \\
\hline Per 1-year increase (continuous variable) & 0.95 & 0.83-1.09 & 0.97 & $0.84-1.10$ \\
\hline \multicolumn{5}{|l|}{ Nulliparous } \\
\hline No & 1.0 (ref) & & 1.0 (ref) & \\
\hline Yes & 1.3 & $0.6-3.0$ & 1.3 & $0.6-3.1$ \\
\hline \multicolumn{5}{|l|}{ Parity, number of births } \\
\hline 0 & 1.0 (ref) & & $1.0(\mathrm{ref})$ & \\
\hline 1 & 1.5 & $0.6-4.0$ & 1.5 & $0.5-4.5$ \\
\hline 2 & 0.8 & $0.3-1.9$ & 0.8 & $0.3-2.1$ \\
\hline 3 & 0.6 & $0.3-1.6$ & 0.6 & $0.2-1.8$ \\
\hline$>3$ & 0.6 & $0.2-1.6$ & 0.6 & $0.2-1.8$ \\
\hline Per additional birth (continuous variable) & 0.81 & $0.67-0.99$ & 0.75 & $0.56-0.99$ \\
\hline \multicolumn{5}{|l|}{$\begin{array}{l}\text { Age at first birth among } 42,844 \\
\text { parous women, years }\end{array}$} \\
\hline$<22$ & 0.8 & $0.4-1.6$ & 0.8 & $0.4-1.8$ \\
\hline $22-25$ & 1.0 (ref) & & 1.0 (ref) & \\
\hline $26-29$ & 0.9 & $0.5-1.6$ & 0.9 & $0.3-2.3$ \\
\hline$>29$ & 1.6 & $0.8-3.2$ & 1.2 & $0.6-2.6$ \\
\hline Per 1-year increase (continuous variable) & 1.02 & $0.96-1.09$ & 0.99 & $0.92-1.06$ \\
\hline \multicolumn{5}{|l|}{ Breastfeeding among 42,844 parous women } \\
\hline No & 1.0 (ref) & & $1.0(\mathrm{ref})$ & \\
\hline Yes & 0.9 & $0.5-1.7$ & 1.0 & $0.5-1.9$ \\
\hline \multicolumn{5}{|l|}{ Use of exogenous hormones } \\
\hline No & 1.0 (ref) & & $1.0(\mathrm{ref})$ & \\
\hline Yes & 0.9 & $0.5-1.8$ & 0.9 & $0.5-1.7$ \\
\hline \multicolumn{5}{|l|}{ Menopausal status at enrollment } \\
\hline Premenopausal & 1.0 (ref) & & $1.0(\mathrm{ref})$ & \\
\hline Postmenopausal (natural) & 0.8 & $0.5-1.4$ & 0.8 & $0.5-1.4$ \\
\hline Height, per $10 \mathrm{~cm}$ increase (continuous variable) & 1.06 & $0.71-1.57$ & 1.03 & $0.68-1.55$ \\
\hline \multicolumn{5}{|l|}{ Body mass index, $\mathrm{kg} / \mathrm{m}^{2}$ at enrollment } \\
\hline$<18.5$ & 0.7 & $0.2-3.0$ & 0.7 & $0.2-2.9$ \\
\hline $18.5-19.9$ & 1.0 & $0.5-2.3$ & 1.0 & $0.4-2.2$ \\
\hline $20-22.9$ & 1.0 (ref) & & 1.0 (ref) & \\
\hline $23-24.9$ & 1.2 & $0.7-2.0$ & 1.2 & $0.7-2.0$ \\
\hline $25-29.9$ & 0.9 & $0.6-1.7$ & 1.0 & $0.5-1.7$ \\
\hline$>29.9$ & 0.8 & $0.2-3.3$ & 0.8 & $0.2-3.3$ \\
\hline Per BMI unit increase (continuous variable) & 1.00 & $0.93-1.07$ & 1.00 & $0.94-1.08$ \\
\hline \multicolumn{5}{|l|}{ Smoking status } \\
\hline Never & 1.0 (ref) & & 1.0 (ref) & \\
\hline Ever & 1.0 & $0.4-2.3$ & 0.9 & $0.4-2.1$ \\
\hline \multicolumn{5}{|l|}{$\begin{array}{l}\text { Exposure to second-hand smoke } \\
\text { among } 41,957 \text { never smokers }\end{array}$} \\
\hline No & 1.0 (ref) & & $1.0(\mathrm{ref})$ & \\
\hline Yes & 1.3 & $0.8-2.1$ & 1.3 & $0.8-2.0$ \\
\hline
\end{tabular}


Table II. Continued.

\begin{tabular}{|c|c|c|c|c|}
\hline \multirow[t]{2}{*}{ Characteristics } & \multicolumn{2}{|c|}{ Age and study area adjusted } & \multicolumn{2}{|c|}{ Multivariable adjustment } \\
\hline & $\mathrm{HR}^{\mathrm{b}}$ & $95 \% \mathrm{CI}$ & $\mathrm{HR}^{\mathrm{c}}$ & $95 \% \mathrm{CI}$ \\
\hline \multicolumn{5}{|l|}{ Alcohol consumption among $44,408^{\mathrm{d}}$} \\
\hline No & 1.0 (ref) & & & \\
\hline Yes & 1.0 & $0.6-1.8$ & 1.0 & $0.5-1.8$ \\
\hline Grams/week (continuous variable) & 1.00 & $0.99-1.00$ & 1.00 & $0.99-1.00$ \\
\hline \multicolumn{5}{|l|}{ Physical activity during leisure time } \\
\hline No & 1.0 (ref) & & 1.0 (ref) & \\
\hline Yes & 1.1 & $0.7-1.8$ & 1.1 & $0.6-1.7$ \\
\hline \multicolumn{5}{|l|}{ Usual sleep duration, $\mathrm{h} /$ day } \\
\hline$<6$ & 1.0 (ref) & & 1.0 (ref) & \\
\hline $6-7$ & 0.5 & $0.2-1.1$ & 0.5 & $0.2-1.0$ \\
\hline$>7$ & 0.4 & $0.2-0.9$ & 0.4 & $0.2-0.9$ \\
\hline Per hour (continuous variable) & 0.80 & $0.65-0.99$ & 0.80 & $0.65-0.99$ \\
\hline \multicolumn{5}{|l|}{$\begin{array}{l}\text { Family history of cancer in first-degree } \\
\text { relative }\end{array}$} \\
\hline No & 1.0 (ref) & & 1.0 (ref) & \\
\hline Yes & 1.5 & $0.9-2.3$ & 1.4 & $0.9-2.3$ \\
\hline
\end{tabular}

(i.e., not excluding missing values from all covariables) were no different than the results of the main models presented in Table II (data not shown).

\section{Discussion}

In this population-based prospective cohort of Japanese women, we found an inverse association between parity and usual sleep duration of more than $7 \mathrm{~h}$ and epithelial ovarian cancer risk. We did not observe any clear associations with other reproductive or menstrual characteristics, body size, or lifestyle characteristics, such as use of exogenous hormones, smoking status, exposure to second-hand smoke, alcohol consumption and physical activity during leisure time. There was an indication of a weak positive association between family history of cancer in a firstdegree relative and epithelial ovarian cancer risk.

The present study has some advantages. The prospective study design potentially avoids selection bias and recall bias, and the relatively high response rate $(>80 \%)$ and very low rate of loss to follow-up indicate that our results well reflect the epithelial ovarian cancer risk profile in the Japanese female population. It is the largest prospective population-based cohort study conducted in Japan to-date that is able to examine risk factors for epithelial ovarian cancer in detail. Nonetheless, the number of incident epithelial ovarian cancer cases in the cohort is limited, due to the relatively low overall incidence of ovarian cancer in both the Japanese population in recent years (SIR 8 per 100,000 women) (1), and in the cohort itself (SIR 11 per 100,000 women). As a result, this study had restricted statistical power in order to detect possible weak associations, which were expected based on information from previously published studies. Moreover, we could not perform stratified analyses by histological subtypes of epithelial ovarian cancer due to lack of statistical power for subgroup analysis.

Information on hysterectomy and oophorectomy was not available for the cohort, and we excluded women who reported artificial menopause at cohort enrollment from all analyses, as the cause (which could have been hysterectomy or oophorectomy) was unknown. Nevertheless, our sensitivity analyses, which did include women who reported artificial menopause before cohort enrollment, yielded results that were similar to those in the analyses excluding them. Finally, several variables had a substantial amount of missing values, and we handled this problem by performing the analysis twice, with and without the inclusion of women and variables with missing values, with virtually identical results.

In our study there was an inverse association between epithelial ovarian cancer risk and increasing number of births (multivariable HR=0.75, 95\% CI 0.56-0.99). Studies carried out elsewhere (9), as well as a few case-control studies in Japan $(8,20,23,25)$ have reported similar inverse associations.

Previous studies, mainly from Western countries, have indicated an association between nulliparity, and, less consistently, early age at menarche, delayed age at first birth, never breastfeeding, and ovarian cancer risk (9). In addition, one casecontrol study in Japan reported a decrease in ovarian cancer risk with older age at menarche (25). However, we could not confirm these findings; none of these associations were statistically significant in our study. This could be due to a different epithelial ovarian cancer risk profile among Japanese women compared to women in North America and Europe, where most previous studies were performed. Indeed, the mean age 
at menarche in our cohort was higher than among Caucasians. Alternatively, it could be explained by the limited statistical power we used in order to detect relatively weak associations.

In our study no associations were observed between exogenous hormone use and epithelial ovarian cancer risk. The International Agency for Research on Cancer (IARC) recently evaluated the evidence on the carcinogenicity of pharmaceuticals to humans, and concluded that use of combined estrogenprogestin oral contraceptives decreases ovarian cancer risk, while estrogen-only HRT increases risk (10). In our study the information on use of exogenous hormones was collected as a single variable, grouping together oral contraceptives and HRT, which is an important limitation. Thus the fact that we did not find any effect is not surprising, as the opposite effects of oral contraceptives and HRT could in theory cancel each other out. Results from a sensitivity analysis removing this variable entirely from the multivariate models did not substantially alter risk estimates for the other variables analyzed. Moreover, reported exogenous hormone use in our study was most likely due to oral contraceptive use, as HRT use in Japan is extremely low. Indeed, HRT use was reported by only $12 \%$ of our study population, among women with and without epithelial ovarian cancer alike, and the duration of use was usually extremely short ( $>1$ year for the entire cohort and for women with epithelial ovarian cancer). This proportion of women and short length of use is far lower than those reported in studies from the United States and Western Europe, from where most of the evidence on associations between exogenous hormones and ovarian cancer emanates. Previous case-control studies in Japan reported either an inverse association between ovarian cancer risk and oral contraceptive use $(18,23)$, or no association $(20,25)$.

Menopausal status at cohort enrollment was not associated with epithelial ovarian cancer risk in our study. Also there was no statistically significant association between height and epithelial ovarian cancer risk. According to the World Cancer Research Fund (WCRF), adult attained height probably increases ovarian cancer risk. Although it is considered that height per se is unlikely to directly modify cancer risk, it may be a marker for genetic, environmental, hormonal and nutritional factors affecting growth from preconception to completion of linear growth (13). The evidence on which the WCRF based its evaluation emanated from 12 studies: nine in Western populations and three in Asian populations. Among the studies in Asian populations, two were case-control studies from Japan, both of which found a non-statistically significant increase in relative risk per $10 \mathrm{~cm}$ increase in height: Mori et al (8) reported an odds ratio (OR) of 1.11 (0.67-1.88) and Hirose et al (24) an OR of 1.12 (0.67-1.88), and one casecontrol study in China reported an OR of 1.26 (0.88-1.80) (32). Again, the mean stature of women in our study was lower than in other studies, including those on which the WCRF based its conclusions. We found no clear association between BMI and epithelial ovarian cancer risk in our study; the WCRF concluded that there was limited evidence, and that no conclusion could be reached about any relationship between body fatness, abdominal fatness, weight change, or energy intake (which we could not evaluate in our study) and ovarian cancer risk (13). The mean BMI in our cohort was also lower than in the studies included in the WCRF evaluation. One previous
Japanese cohort study (14) and three Japanese case-control studies $(8,23,24)$ reported a positive association between increasing BMI and risk for epithelial ovarian cancer, although the results were not entirely consistent.

The prevalence of smoking in our cohort was very low, and there was no evidence of an association with epithelial ovarian cancer risk. As most of the women who had ever smoked in our cohort were former smokers, we did not perform separate analyses for current smokers, as our statistical power was too low to reach any conclusions. Although most studies in Western populations have not indicated an association between smoking and ovarian cancer risk, some recent studies, including cohort studies, have reported a positive association between smoking and risk of mucinous ovarian cancer (12). A recent comprehensive review of the worldwide scientific literature on the carcinogenicity of tobacco smoke (12) concluded that there was sufficient evidence to classify cigarette smoking as carcinogenic in relation to mucinous ovarian cancer, but not in relation to other histological subtypes. One previous cohort study in Japan reported a positive association between current smokers of 10-19 cigarettes per day, compared to never smokers, and ovarian cancer risk (15).

Exposure to second-hand smoke was over $30 \%$ among women in our cohort, most probably reflecting the high prevalence of smoking among Japanese men. We found no association between exposure to second-hand smoke and risk of epithelial ovarian cancer in our study, in agreement with the scientific literature (12). There were an insufficient number of studies on exposure to second-hand smoke and epithelial ovarian cancer risk in the scientific literature to allow the IARC to draw conclusions about any possible association (12). We could not identify in the literature any previous study about second-hand smoke and ovarian cancer risk in Japan.

Alcohol consumption was not positively associated with epithelial ovarian cancer risk in our cohort, in agreement with most of the scientific literature (12), save one Japanese case-control study which reported an inverse association (21). Detailed analysis by type of beverage (beer, sake, etc.) was not possible in our cohort due to small numbers, although there is no evidence in the scientific literature suggesting that we should expect a different effect on ovarian cancer risk by type of alcoholic beverage (12).

Physical activity during leisure time was not associated with epithelial ovarian cancer risk in our study. Similarly, the WCRF concluded that there is limited evidence and no conclusions can be drawn based on the published scientific literature on a possible association between physical activity during leisure time and ovarian cancer risk (13).

Our findings of an association between usual sleep duration and epithelial ovarian cancer risk are novel, as we could not identify prospective cohort studies describing such an association previously. We cannot entirely rule out the possibility that this is a chance finding. However, if studies in other populations could confirm these results, this finding may be of public health significance in cancer prevention. A protective effect of usual sleep duration has been suggested for other cancer sites, as well as for cardiovascular diseases $(33,34)$. Mechanisms underlying the suggested association between usual sleep duration and epithelial ovarian cancer risk may include the melatonin pathway (33). Light exposure during biological darkness and 
the associated circadian disruption seem to affect cancer incidence and growth in the majority of studies (46 out of 56 studies) in animal models, which were evaluated recently by the IARC (33). For ovarian cancer in particular, studies in animal models indicated that pinealectomized hamsters that were inoculated subcutaneously with ovarian tumor cells had an ovarian tumor volume that was about five-fold greater than control hamsters with intact pineal glands (35). Pinealectomy suppresses the physiological nocturnal melatonin signal leading to the enhancement of cancer development and/or growth in animal models. At the same time, this procedure indirectly raises the question of whether the physiological nocturnal melatonin signal from the pineal gland inhibits the process of tumorigenesis in animal models (33).

Family history of cancer in a first-degree relative was not statistically significantly associated with epithelial ovarian cancer risk in our study, as has been reported in studies elsewhere (9), and in a few Japanese case-control studies $(8,20,23)$. However, the HR point estimate indicated a weak increase in epithelial ovarian cancer risk among women with a family history of cancer in a first-degree relative. The number of cases in our study was too limited to perform analyses by cancer type in the relatives, which would be desirable. Further follow-up of the cohort is needed for these types of detailed analyses.

In conclusion, in this prospective cohort study on epithelial ovarian cancer in Japanese women, parity and usual sleep duration of more than $7 \mathrm{~h}$ per day were both associated with a decrease in risk. Other risk factors that were reported to be associated with epithelial ovarian cancer in several studies in Western countries (9), a few Japanese case-control studies (8,18-20,23-25), and one Japanese cohort study $(14,15)$, such as age at menarche, age at first birth, breastfeeding, use of exogenous hormones, height, BMI and physical activity, were not observed in this study in Japan. Family history of ovarian cancer was positively associated with the HR point estimate (although not significantly), in agreement with studies among Western women and a few Japanese case-control studies $(8,20,23)$, although the association in our study was not statistically significant. Smoking or exposure to second-hand smoke, as well as alcohol consumption, were not associated with epithelial ovarian cancer risk in our study, which was also the case in most studies among Western women. Previous prospective cohort studies in Japan indicated a possible association with smoking (15) and BMI (14), although it was not statistically significant. Overall, the previous Japanese case-control studies presented mixed results regarding different risk factors for ovarian cancer $(8,18-25)$.

\section{Acknowledgements}

The authors thank all staff members in each study area and the central offices for their cooperation and technical assistance; the Iwate Aomori, Ibaraki, Niigata, Osaka, Kochi, Nagasaki, and Okinawa Cancer registries for providing incidence data; Dr Reiko Suzuki for helpful comments in early drafts of the manuscript; Mrs Margrethe Meo for secretarial assistance; and Mrs Trudy Perdrix-Thoma for editorial assistance and language review. This study received financial support from the Japanese Government: National Cancer Center Research and Development Fund and the Ministry of Health, Labor and Welfare of Japan: Grant in Aid for Cancer Research and Third Term Comprehensive Control Research for Cancer. Dr E. Weiderpass was supported by a visiting scientist award from the Foundation for Promotion of Cancer Research of Japan.

\section{Study Group members}

Members of the Japan Public Health Center-based Prospective Study Group (principal investigator: S. Tsugane) are: S. Tsugane, M. Inoue, T. Sobue, and T. Hanaoka, National Cancer Center, Tokyo; J. Ogata, S. Baba, T. Mannami, A. Okayama, and Y. Kokubo, National Cardiovascular Center, Osaka; K. Miyakawa, F. Saito, A. Koizumi, Y. Sano, I. Hashimoto, T. Ikuta and Y. Tanaba, Iwate Prefectural Ninohe Public Health Center, Iwate; Y. Miyajima, N. Suzuki, S. Nagasawa, Y.Furusugi, N. Nagai and Y. Ito, Akita Prefectural Yokote Public Health Center, Akita; H. Sanada, Y. Hatayama, F. Kobayashi, H. Uchino, Y. Shirai, T. Kondo, R. Sasaki, Y. Watanabe, Y. Miyagawa, Y. Kobayashi and M. Machida, Nagano Prefectural Saku Public Health Center, Nagano; Y. Kishimoto, E. Takara, T. Fukuyama, M. Kinjo, M. Irei, and H. Sakiyama, Okinawa Prefectural Chubu Public Health Center, Okinawa; K.Imoto,H. Yazawa, T. Seo, A. Seiko, F. Ito, F. Shoji and R. Saito, Katsushika Public Health Center, Tokyo; A. Murata, K. Minato, K. Motegi, T. Fujieda and S. Yamato, Ibaraki Prefectural Mito Public Health Center, Ibaraki; K. Matsui, T. Abe, M. Katagiri, and M. Suzuki, Niigata Prefectural Kashiwazaki and Nagaoka Public Health Center, Niigata; M. Doi, A. Terao, Y. Ishikawa, and T. Tagami, Kochi Prefectural Chuo-higashi Public Health Center, Kochi; H. Sueta, H. Doi, M. Urata, N. Okamoto, F. Ide and H. Goto, Nagasaki Prefectural Kamigoto Public Health Center, Nagasaki; H. Sakiyama, N. Onga, H. Takaesu, and M. Uehara, Okinawa Prefectural Miyako Public Health Center, Okinawa; F. Horii, I. Asano, H. Yamaguchi, K. Aoki, S. Maruyama, M. Ichii, and M. Takano, Osaka Prefectural Suita Public Health Center, Osaka; Y. Tsubono, Tohoku University, Miyagi; K. Suzuki, Research Institute for Brain and Blood Vessels Akita, Akita; Y. Honda, K. Yamagishi, S. Sakurai and N. Tsuchiya, Tsukuba University, Ibaraki; M. Kabuto, National Institute for Environmental Studies, Ibaraki; M. Yamaguchi, Y. Matsumura, S. Sasaki, and S. Watanabe, National Institute of Health and Nutrition, Tokyo; M. Akabane, Tokyo University of Agriculture, Tokyo; T. Kadowaki, Tokyo University, Tokyo; M. Noda and T. Mizoue, National Center for Global Health and Medicine, Tokyo; Y. Kawaguchi, Tokyo Medical and Dental University, Tokyo; Y. Takashima and Y. Yoshida, Kyorin University, Tokyo; K. Nakamura, Niigata University, Niigata; S. Matsushima and S. Natsukawa, Saku General Hospital, Nagano; H. Shimizu, Sakihae Institute, Gifu; H. Sugimura, Hamamatsu University, Shizuoka; S. Tominaga, Aichi Cancer Center, Aichi; N. Hamajima, Nagoya University, Aichi; H. Iso, Osaka University, Osaka; M. Iida, W. Ajiki, and A. Ioka, Osaka Medical Center for Cancer and Cardiovascular Disease, Osaka; S. Sato, Chiba Prefectural Institute of Public Health, Chiba; E. Maruyama, Kobe University, Hyogo; M. Konishi, K. Okada, and I. Saito, Ehime University, Ehime; N. Yasuda, Kochi University, Kochi; S. Kono, Kyushu University, Fukuoka; S. Akiba, Kagoshima University, Kagoshima. 


\section{References}

1. Matsuda T, Marugame T, Kamo K, Katanoda K, Ajiki W, Sobue T and the Japan Cancer Surveillance Research Group: Cancer incidence and incidence rates in Japan in 2004: based on data from 14 population-based cancer registries in the Monitoring of Cancer Incidence in Japan (MCIJ) Project. Jpn J Clin Oncol 40: 1192-1200, 2010

2. Ferlay J, Bray F, Pisani P and Parkin DM: GLOBOCAN 2002: Cancer incidence, mortality and prevalence worldwide, Version 2.0 (CD-ROM). International Agency for Research on Cancer, Lyon, 2004.

3. Ferlay J, Shin HR, Bray F, Forman D, Mathers C and Parkin DM: Estimates of worldwide burden of cancer in 2008: GLOBOCAN 2008. Int J Cancer 127: 2893-2917, 2010.

4. Tamakoshi K, Kondo T, Yatsuya H, Hori Y, Kikkawa F and Toyoshima H: Trends in the mortality (1950-1997) and incidence (1975-1993) of malignant ovarian neoplasm among Japanese women: analyses by age, time, and birth cohort. Gynecol Oncol 83: 64-71, 2001.

5. Qiu S, Katanoda K, Marugame T and Sobue T: A joinpoint regression analysis of long-term trends in cancer mortality in Japan (1958-2004). Int J Cancer 124: 443-448, 2009.

6. Herrinton LJ, Stanford JL, Schwarts SM and Weiss NS: Ovarian cancer incidence among Asian migrants to the United States and their descendants. J Natl Cancer Inst 86: 1336-1339, 1994.

7. Kamo KI and Sobue T: Mortality trend of prostate, breast, uterus, ovary, bladder and kidney and other urinary tract cancer in Japan by birth cohort. Jpn J Clin Oncol 34: 561-563, 2004.

8. Mori M,Nishida T, Sugiyama T, Komai K, Yakushiji M,Fukuda K, Tanaka T, Yokoyama M and Sugimori H: Anthropometric and other risk factors for ovarian cancer in a case-control study. Jpn J Cancer Res 89: 246-253, 1998

9. Permuth-Wey J and Sellers TA: Epidemiology of ovarian cancer In: Cancer Epidemiology. Vol 2. Modifiable Factors. Methods of Molecular Biology 472. Verma M (ed.). Humana Press, Totowa, pp413-437, 2009.

10. Grosse Y, Baan R, Straif K, Secretan B, El Ghissassi F, Bouvard V, Benbrahim-Tallaa L, Guha N, Galichet L and Cogliano V; for the WHO International Agency for Research on Cancer Monograph Working Group: A review of human carcinogens-Part A: pharmaceuticals. Lancet Oncol 10: 13-14, 2009.

11. Bevier M, Sundquist J and Hemminki K: Does the time interval between first and last birth influence the risk of endometrial and ovarian cancer? Eur J Cancer 47: 586-591, 2011.

12. Secretan B, Straif K, Baan R, Grosse Y, El Ghissassi F, Bouvard V, Benbrahim-Tallaa L, Guha N, Freeman C, Galichet L and Cogliano V; for the WHO International Agency for Research on Cancer Monograph Working Group: A review of human carcinogens-Part E: tobacco, areca nut, alcohol, coal smoke, and salted fish. Lancet Oncol 10: 1033-1034, 2009.

13. World Cancer Research Fund, American Institute for Cancer Research. Food, Nutrition, Physical Activity, and the Prevention of Cancer: A Global Perspective. AICR, Washington DC, 2007.

14. Niwa Y, Yatsuya H, Tamakoshi K, Nishio K, Kondo T, Lin Y, Suzuki S, Wakai K, Tokudome S, Yamamoto A, Hamajima N, Toyoshima H, Tamakoshi A and the JACC Study Group: Relationship between body mass index and the risk of ovarian cancer in the Japanese population: findings from the Japanese Collaborate Cohort (JACC) study. J Obstet Gynaecol Res 31: 452-458, 2005.

15. Niwa Y, Wakai K, Suzuki S, Tamakoshi K, Lin Y, Yatsuya H, Kondo T, Nishio K, Yamamoto A, Tokudome S, Hamajima N, Toyoshima H, Tamakoshi $\mathrm{H}$ and the JACC Study Group: Cigarette smoking and the risk of ovarian cancer in the Japanese population: Findings from the Japanese Collaborate Cohort study. J Obstet Gynaecol Res 31: 144-151, 2005.

16. Sakauchi F, Khan MMH, Mori M, Kubo T, Fujino Y, Suzuki S, Tokudome S, Tamakoshi A and the JACC Study Group: Dietary habits and risk of ovarian cancer death in a large-scale cohort study (JACC Study) in Japan. Nutr Cancer 57: 138-145, 2007.
17. Inoue M, Iwasaki M, Otani T, Sasazuki S, Noda $M$ and Tsugane S; for the Japan Public Health Center-based Prospective Study Group: Diabetes mellitus and the risk of cancer: results from a large-scale population-based cohort study in Japan. Arch Intern Med 166: 1871-1877, 2006.

18. Mori M, Kiyosawa $\mathrm{H}$ and Miyake H: Case-control study of ovarian cancer in Japan. Cancer 53: 2746-2752, 1984.

19. Mori $\mathrm{M}$ and Miyake $\mathrm{H}$ : Dietary and other risk factors of ovarian cancer among elderly women. Jpn J Cancer Res 79: 997-1004, 1988.

20. Mori M, Harabuchi I, Miyake H, Casagrande JT, Henderson BE and Ross RK: Reproductive, genetic, and dietary risk factors for ovarian cancer. Am J Epidemiol 128: 771-777, 1988.

21. Kato I, Tominaga S and Terao C: Alcohol consumption and cancers of hormone-related organs in females. Jpn J Clin Oncol 19: 202-207, 1989.

22. Kodama M, Kodama T, Totani R, Suzuoki Y and Kodama M: Relation between the hormonal and epidemiological aspects of ovarian cancer patients in Japan. Anticancer Res 12: 1593-1602, 1992.

23. Mori M, Nishimura H, Nishida T, Sugiyama T, Komai K, Ide H, Yakushiji M, Fukuda K, Tanaka T, Yokoyama M and Sugimori H: A case-control study of ovarian cancer to identify its risk factors. Nippon Sanka Fujinka Gakkai Zasshi 48: 875-882, 1996 (In Japanese).

24. Hirose K, Tajima K, Hamajima N, Kuroishi T, Kuzuya K, Miura $\mathrm{S}$ and Tokudome S: Comparative case-referent study of risk factors among hormone-related female cancers in Japan. Jpn J Cancer Res 90: 255-261, 1999.

25. Fujita M, Tase T, Kakugawa Y, Hoshi S, Nishino Y, Nagase S, Ito K, Niikura H, Yaegashi N and Minami Y: Smoking, earlier menarche and low parity as independent risk factors for gynecologic cancers in Japanese: a case-control study. Tohoku J Exp Med 216: 297-307, 2008.

26. Weiss NS, Cook LS, Farrow DC and Rosenblatt KA: Ovarian cancer. In: Cancer Epidemiology and Prevention. 2nd edition. Schottenfeld D and Fraumeni JF (eds.) Oxford University Press, New York, pp1040-1057, 1996.

27. Korn EL, Graubard BI and Midthune D: Time-to-event analysis of longitudinal follow-up of a survey: choice of the time-scale. Am J Epidemiol 145: 72-80, 1997.

28. Grambsch P and Therneau T: Proportional hazards tests and diagnostics based on weighted residuals. Biometrika 81: 515-526, 1994.

29. Tsugane S, Sasaki S and Tsubono Y: Under- and overweight impact on mortality among middle-aged Japanese men and women. A 10-y follow-up of the JPHC Study Cohort I. Int J Obes 26: 529-537, 2002

30. Iwasaki M, Otani T, Inoue $M$, Sasazuki S and Tsugane $S$; for the Japan Public Health Center-based Prospective Study Group: Body size and risk for breast cancer in relation to estrogen and progesterone receptor status in Japan. Ann Epidemiol 17: 304-312, 2007.

31. Iwasaki M, Otani T, Inoue M, Sasazuki S and Tsugane S; for the Japan Public Health Center-based Prospective Study Group: Role and impact of menstrual and reproductive factors on breast cancer risk in Japan. Eur J Cancer Prev 16: 116-123, 2007.

32. Zhang M, Xie X, Lee AH, Binns CW and Holman CDJ. Body mass index in relation to ovarian cancer survival. Cancer Epidemiol Biomarkers Prev 14: 1307-1310, 2005.

33. International Agency for Research on Cancer: IARC Monographs on the Evaluation of Carcinogenic Risks to Humans Volume 98: Painting, Firefighting, and Shiftwork. International Agency for Research on Cancer, Lyon, 2010.

34. Cappuccio FP, Cooper D, D'Elia L, Strazzullo P and Miller MA: Sleep duration predicts cardiovascular outcomes: a systematic review and meta-analysis of prospective studies. Eur Heart J 32: 1484-1492, 2011

35. Das Gupta TK: Influence of the pineal gland on the growth and spread of malignant tumors. Surg Forum 19: 83-84, 1968. 